\title{
Timing of Elective Termination of Pregnancy in Preterm Premature Rupture of Membranes : Prospective Randomized Controlled Trial
}

\section{Original Article}

\author{
Mohamed A. Abdel-Hafeez, Sabry S. Hasan, Mohammed M. Abdel-Mawgood, \\ Ahmed M. Essam El-Dein
}

Department of Obstetrics and Gynecology, Faculty of Medicine, Ain-Shams University, Cairo

\begin{abstract}
Background: Rupture of membranes is associated with high incidence of maternal and fetal morbidities as well as the neonatal morbidity and mortality resulting from prematurity if preterm birth ensues.

Aim: This study aimed to evaluate maternal and neonatal outcomes of elective delivery at 34 versus 36 weeks of gestations in pregnancies complicated by preterm premature rupture of membranes and had been managed expectantly.

Materials and Methods: This is a prospective randomized controlled trial which was conducted at Ain Shams University Maternity hospital between March and August 2019. 140 women pregnant between 30 and 32 weeks and diagnosed with preterm premature rupture of membranes were admitted to receive conservative management and then they were randomly divided into 2 equal groups regarding time of elective delivery either late delivery at 36 weeks or early delivery at 34 weeks.

Results: The incidence of intrauterine infections was comparable between both groups. The rate of chorioamnionits was $8.6 \%$ in the late delivery group versus $4.3 \%$ in the early delivery group while endometritis was diagnosed in $2.9 \%$ in the late delivery group and $1.4 \%$ in the early delivery group. The incidence of spontaneous onset of preterm labor, cord prolapse and placental abruption was similar in both groups. Neonates of the late delivery group had significantly more Apgar score at 5 minutes $(6.4 \pm 1.0$ versus $5.0 \pm 0.9, p<0.001)$ and more birth weight $(2388.6 \pm 240.3$ versus $2173.7 \pm 231.5$, $p<0.001)$ in addition to significantly less incidence of respiratory distress syndrome $(2.9 \%$ versus $11.4 \%, p=0.049)$ and fewer neonatal intensive care unit admissions $(27.1 \%$ versus $54.3 \%, p=0.002)$. The frequency of neonatal infections was higher in the late delivery group but this difference was not significant statistically $(5.7 \%$ versus $2.9 \%, p>0.05)$.

Conclusion: Early delivery at 34 weeks does not reduce the rate of chorioamnionitis, but does increase the likelihood of respiratory distress syndrome and the rate of neonatal intensive care unit admissions. Our findings support the practice of conservative management in pregnant women with ruptured membranes if there is no contraindication to extending the pregnancy.
\end{abstract}

Key Words: Chorioamnionitis, neonatal outcome, preterm, prematurity, rupture of membranes.

Received: 04 August 2021, Accepted: 14 August 2021

Corresponding Author: Mohamed A. Abdel-Hafeez, Department of Obstetrics and Gynecology, Faculty of Medicine, Ain-Shams University, Cairo, Egypt, Tel.: +20 1069491030, E-mail: abdelhafeezmohamed014@gmail.com

ISSN: 2090-7265, August 2021, Vol.11, No. 3

\section{INTRODUCTION}

Rupture of fetal membranes is one of the common obstetrics conditions with an incidence of approximately $3 \%$ of all pregnancies and preterm premature rupture of membranes (PPROM) precedes around one third of preterm births ${ }^{[1]}$. The term preterm refers to rupture of the membranes before completed 37 weeks of gestation and the term premature refers to rupture of the membranes before the onset of labor. The etiology of PPROM remains unknown but several risk factors have been reported in the literature with urogenital infection, smoking during the current pregnancy and cervical weakness being the most consistent reported risk factors ${ }^{[2,3]}$.

Pregnancies complicated by PPROM are considered high risk due to high incidence of maternal and fetal morbidities as well as the neonatal morbidity and mortality resulting from prematurity if preterm birth ensues ${ }^{[4]}$. Gestational age at delivery is the main factor affecting the outcome of pregnancies complicated by PPROM and expectant management with antibiotics and corticosteroids to enhance fetal maturity is the recommended management for women presenting with PPROM before 34 weeks if continuing pregnancy is not contraindicated ${ }^{[5,6]}$. The mainstay in the management is strict follow up with combined clinical and lab assessment which is recommended for early detection and proper intervention if intrauterine infection complicates the condition ${ }^{[5]}$.

The decision about timing of delivery in women with PPROM is still in debate. Early studies recommended delivery by 34 weeks of gestation to minimize the risk of infection ${ }^{[5]}$ but a similar risk of infection with better 
neonatal outcomes have been reported in a Cochrane review if delivery is planned at 37 weeks $^{[7]}$.

\section{AIM OF THE WORK}

This work aimed to evaluate maternal and neonatal outcomes of elective delivery at 34 versus 36 weeks of gestations in pregnancies complicated by preterm premature rupture of membranes and had been managed expectantly.

\section{PATIENTS AND METHODS}

This study was a prospective randomized controlled study which was conducted at the department of Obstetrics and Gynecology, Ain Shams University Maternity Hospital from March to August 2019. The study included 140 women with a diagnosis of preterm premature rupture of membrane presenting between 30 and 32 weeks of gestation. The study was approved by the local Institutional Review Board of obstetrics and gynecology department and all participants signed an informed consent before inclusion.

Women with singleton gestations between 30weeks and 32 weeks of gestation complicated by preterm premature rupture of the membranes were eligible for this study and we excluded women with fetal compromise, labor on admission, and medical or obstetric complications (such as suspected chorioamnionitis, hypertensive disorders, diabetes mellitus, active genital herpes, placenta previa, infection, meconium-stained fluid, or severe fetal anomalies). Gestational age was confirmed by a reliable last menstrual period and/or early sonogram and amniorrhexis was confirmed by history and visualization of pooling fluid in the posterior vaginal fornix through the cervix during sterile speculum examination. No digital examination was performed in any patient.

After inclusion the patients were randomly assigned to either late delivery at 36 weeks or early delivery at 34 weeks of gestation. All women in both groups were managed with conservative management which according to the hospital protocol consists of admission to the hospital and administration of antibiotics and corticosteroids for enhancing lung maturity as well as close maternal and fetal surveillance till the planned time for elective delivery.

Antibiotic prophylaxis was given as Azithromycin 1 gram orally upon admission, PLUS Ampicillin $2 \mathrm{~g}$ intravenously every 6 hours for 48 hours, followed by Amoxicillin $500 \mathrm{mg}$ orally three times daily or $875 \mathrm{mg}$ orally twice daily for an additional five days. Corticosteroids course was administered as a single course of dexamethasone $6 \mathrm{mg}$ intramuscular injection every 12 hours for four times.

Chorioamnionitis diagnosis was based on combination of maternal symptoms and examination in addition to fetal heart rate monitoring ${ }^{[5]}$. endometritis was suspected if there was postpartum pyrexia and suspected cases were subjected to transvaginal ultrasound and diagnosis was confirmed if the scan revealed turbid collection in endometrial cavity and those patients were managed by antibiotics according to the hospital protocol.

Delivery was planned by induction of labor and cesarean section was performed for standard indications. In the absence of contraindications to labor and vaginal birth, digital cervical examination was carried out to determine the status of the cervix and labor was induced by oxytocin or prostaglandins per the hospital protocol according to the favorability of the cervix for induction of labor.

The primary outcome of this study was the incidence of intrauterine infection and secondary outcomes were the maternal and neonatal outcome.

Random list was previously achieved using a computergenerated sequence through Research Randomizer (www randomizer.org) and sequentially numbered opaque sealed envelopes (SNOSE) method was used to conceal the group before patients' assignment.

\section{STATISTICAL ANALYSIS}

Quantitative variables were expressed as mean and standard deviation and analysis were done using Student $t$ test and qualitative variables were expressed as frequencies and percents and analysis were done using Chi square test. Analysis for this study was done with intention to prevent analysis and relative risk with its $95 \%$ confidence interval was calculated where possible.

\section{RESULTS}

This study included 140 pregnant women between 30 and 32 weeks of gestation and diagnosed to have PPROM. All women were managed conservatively and were randomly divided into 2 equal groups: late versus early delivery. The mean of gestational age at inclusion was $31.4 \pm 0.8$ weeks in the late delivery group compared to $31.2 \pm 0.6$ weeks in the early delivery group which was insignificant statistically, other demographic data were comparable between both groups and are presented in (Table 1).

Table 1: demographic characteristics of the studied patients among late compared to early delivery groups

\begin{tabular}{lcc}
\hline Character & $\begin{array}{c}\text { late delivery at } \\
37 \text { weeks } \\
(\mathrm{n}=70)\end{array}$ & $\begin{array}{c}\text { early delivery } \\
\text { at } 34 \text { weeks } \\
(\mathrm{n}=70)\end{array}$ \\
\hline age (years) & $27.1 \pm 3.5$ & $27.7 \pm 3.1$ \\
BMI (kg/m2) & $26.3 \pm 1.9$ & $26.7 \pm 2.1$ \\
multipara & $54(77.1 \%)$ & $48(68.6 \%)$ \\
$\begin{array}{l}\text { gestational age at } \\
\text { inclusion (weeks) }\end{array}$ & $31.4 \pm 0.8$ & $31.2 \pm 0.6$ \\
\hline
\end{tabular}

Data presented as mean \pm standard deviation or number (percentage) 
The difference in the incidence of intrauterine infections was comparable between both groups. The rate of chorioamnionits was $8.6 \%$ (6 patients) in the late delivery group versus $4.3 \%$ ( 3 patients) in the early delivery group while endometritis was diagnosed in $2.9 \%$ ( 2 patients) in the late delivery group and $1.4 \%$ (1 patient) in the early delivery group. No cases diagnosed with maternal septicemia in both groups (Table 2).

The incidence of spontaneous onset of preterm labor was $4.3 \%$ (3 patients) versus $2.9 \%$ ( 2 patients) in the late delivery versus early delivery group respectively and this difference was also not significant. The rate of cord prolapse was comparable between groups and no cases of placental abruption was seen in both groups. A comparison regarding the maternal outcome is shown in (Table 2).

The rate of delivery before the assigned time of elective delivery was $20 \%$ (14 patients) in the late delivery group compared to $14.2 \%$ (10 patients) in the early delivery group. The causes were spontaneous onset of labor, chorioamnionits, cord prolapse and suspected antenatal fetal compromise. The difference in the rate of complications required delivery before the assigned elective time was similar between both groups.

Table 3 showed comparison between both groups regarding neonatal outcome. Neonates of the late delivery group had significantly more Apgar score at 5 minutes $(6.4 \pm 1.0$ versus $5.0 \pm 0.9, p<0.001)$ and more birth weight $(2388.6 \pm 240.3$ versus $2173.7 \pm 231.5$, $p<0.001)$ in addition to significantly less incidence of respiratory distress syndrome (RDS) $(2.9 \%$ versus $11.4 \%, p=0.049)$ and fewer neonatal intensive care unit admissions (NICU) $(27.1 \%$ versus $54.3 \%, p=0.002)$. The frequency of neonatal infections was higher in the late delivery group but this difference was not significant statistically $(5.7 \%$ versus $2.9 \%, p>0.05)$.

Table 2: Comparison between late delivery and early delivery groups as regards maternal outcome

\begin{tabular}{|c|c|c|c|c|}
\hline & late delivery $(\mathrm{n}=70)$ & early delivery $(n=70)$ & $\mathrm{RR}(95 \% \mathrm{CI})$ & $p$ \\
\hline Chorioamnionitis & $6(8.6 \%)$ & $3(4.3 \%)$ & $2.00(0.52-7.68)$ & $0.493^{\mathrm{a}}$ \\
\hline Endometrities & $2(2.9 \%)$ & $1(1.4 \%)$ & $2.00(0.19-21.56)$ & $1.000^{\mathrm{a}}$ \\
\hline Septicemia & $0(0.0 \%)$ & $0(0.0 \%)$ & - & -- \\
\hline cesarean section & $20(28.6 \%)$ & $32(45.7 \%)$ & $0.63(0.40-0.98)$ & $0.036^{\mathrm{b}}$ \\
\hline Cord prolapse & $2(2.9 \%)$ & $5(7.1 \%)$ & $0.40(0.08-1.99)$ & $0.441^{\mathrm{a}}$ \\
\hline Placental abruption & $0(0.0 \%)$ & $0(0.0 \%)$ & - & -- \\
\hline spontaneous onset & $3(4.3 \%)$ & $2(2.9 \%)$ & - & $0.681^{\mathrm{a}}$ \\
\hline
\end{tabular}

Data presented as number (percentage), analysis done using a $=$ Fisher's Exact test or $\mathrm{b}=$ Chi square test, $\mathrm{RR}=$ relative risk, $\mathrm{CI}=$ confidence interval

Table 3: comparison between late delivery and early delivery groups as regards neonatal outcome

\begin{tabular}{|c|c|c|c|c|}
\hline & late delivery $(\mathrm{n}=70)$ & early delivery $(\mathrm{n}=70)$ & $\mathrm{RR}(95 \% \mathrm{CI})$ & $p$ \\
\hline APGAR 5 & $6.4 \pm 1.0$ & $5.0 \pm 0.9$ & - & $<0.001 \mathrm{a}$ \\
\hline neonatal sepsis & $4(5.7 \%)$ & $2(2.9 \%)$ & $2.00(0.38-10.57)$ & $0.681^{\mathrm{c}}$ \\
\hline RDS & $2(2.9 \%)$ & $8(11.4 \%)$ & $0.25(0.06-1.14)$ & $0.049^{\mathrm{b}}$ \\
\hline IVH & $1(1.4 \%)$ & $5(7.1 \%)$ & $0.20(0.02-1.67)$ & $0.209^{\mathrm{c}}$ \\
\hline NICU admission & $19(27.1 \%)$ & $38(54.3 \%)$ & $0.50(0.32-0.78)$ & $0.002^{\mathrm{b}}$ \\
\hline estimated fetal weight at inclusion & $1863.5 \pm 177.0$ & $1820.5 \pm 199.7$ & - & $0.179^{\mathrm{a}}$ \\
\hline birth weight & $2388.6 \pm 240.3$ & $2173.7 \pm 231.5$ & - & $<0.001^{\mathrm{a}}$ \\
\hline
\end{tabular}

Data presented as mean \pm standard deviation or number (percentage), analysis done using a $=$ student's $\mathrm{T}$ test, $\mathrm{b}=\mathrm{Chi}$ square or $\mathrm{c}=$ Fisher's Exact test, RR $=$ relative risk, $\mathrm{CI}=$ confidence interval, $\mathrm{IVH}=$ intraventricular hemorrhage, NICU $=$ neonatal intensive care unit, $\mathrm{RDS}=$ respiratory distress syndrome

\section{DISCUSSION}

The best timing for delivery of women diagnosed with PPROM is still in debate. Most recommend delivery in the late preterm period (34-36+6 weeks) based on studies reporting a rise in the frequency of the major adverse outcome resulting from prolonging pregnancy, namely chorioamnionitis ${ }^{[8]}$. This practice has been criticized as more recent studies demonstrate that the incidence of chorioamnionitis is not significantly increased if conservative management is extended to term ${ }^{[5-7,9]}$. Gestational age at delivery is suggested to be the major determinant for adverse neonatal outcome as poor outcomes are related to prematurity rather than to PPROM per $\mathrm{se}^{[9]}$.

This study aimed to find out if the effect of prolonging pregnancy in cases with PPROM is associated with better neonatal outcome balanced to the adverse outcomes that may result from intrauterine infections. We found that late delivery at 36 weeks of gestation significantly reduced the frequency of RDS and NICU admissions and was associated with significantly higher apgar scores at 5 minutes as well as with higher birth rate while there was no significant difference in the frequency of neonatal sepsis. Furthermore, the frequency of maternal intrauterine 
infections was comparable between late and early delivery groups.

In line with these results, a retrospective study analyzing neonatal outcome for singleton pregnancies complicated by PPROM which concluded that early delivery at 34 weeks is associated with problems of prematurity with no improved outcome $^{[9]}$. This was further confirmed in a metaanalysis analyzing 12 trials which agreed to our results in that early delivery by 34 weeks of gestation is associated with poor neonatal outcomes including RDS, mechanical ventilation and NICU admissions while the risk of neonatal sepsis as well as the rate of perinatal mortality was comparable to delayed delivery ${ }^{[7]}$.

On the other hand, an individual patient data metaanalysis ${ }^{[10]}$ comparing immediate delivery versus conservative management in women with PPROM and presenting between 34 and $36+6$ weeks of gestation. The study reported that although adverse neonatal outcomes were comparable, maternal outcome was significantly improved with immediate delivery. The study also reported a significant reduction in the frequency of chorioamnionitis $(\mathrm{RR}=0.21,95 \% \quad \mathrm{CI}=0.13-0.35)$ and antepartum hemorrhage $(\mathrm{RR}=0.57,95 \% \mathrm{CI}=0.34-0.95)$.

In that metaanalysis ${ }^{[10]}$, nearly half of the data was included from a single trial in addition to the significant variation of PPROM to induction of labor interval with heterogenicity in administration of prophylactic antibiotics, tocolytics and antenatal corticosteroids between studies and finally, outpatient management was practiced in a significant proportion of the analyzed data which is not considered a standard management in women with PPROM as advised in the current guidelines ${ }^{[5]}$. This may have influenced that metaanalysis to disagree with current results.

Also in disagreement with the current results, Ramsey et $a l^{[11]}$ reported no neonatal benefit from conservative management beyond 34 weeks of gestation. Schucker et ${ }^{\left[l^{[8]}\right.}$ also reported a significantly more frequent rate of chorioamnionitis with expectant management but they also noted that neonatal sepsis was comparable to immediate delivery.

These earlier studies were carried out before standardization of management of cases diagnosed with PPROM and the effect of standard prophylactic antibiotics should be the major influence on the difference from the more recent reports.

\section{CONCLUSION}

Early delivery at 34 weeks does not reduce rate of chorioamnionitis, but does increase the likelihood of RDS and rate of NICU admission. Our findings support the practice of conservative management in pregnant women with ruptured membranes if there is no contraindication to extending the pregnancy.

\section{CONFLICT OF INTERESTS}

There are no conflicts of interests.

\section{REFERENCES}

1. Tchirikov M, Schlabritz-Loutsevitch N, Maher $\mathrm{J}$, et al. Mid-trimester preterm premature rupture of membranes (PPROM): etiology, diagnosis, classification, international recommendations of treatment options and outcome. Journal of perinatal medicine 2018; 46(5):465-88.

2. Zhou Q, Zhang W, Xu H, Liang H, Ruan Y, Zhou $\mathrm{S}$, Li X. Risk factors for preterm premature rupture of membranes in Chinese women from urban cities. International Journal of Gynecology \& Obstetrics 2014;127(3): 254-9.

3. Mercer BM. (2003): Preterm premature rupture of the membranes. Obstet. Gynecol.; 101(1):178-93.

4. Seelbach-Goebel B. (2013): Antibiotic therapy for premature rupture of membranes and preterm labor and effect on fetal outcome. Geburtshilfe und Frauenheilkunde; 73(12):1218.

5. Thomson AJ, on behalf of the Royal College of Obstetricians and Gynaecologists. Care of women presenting with suspected preterm prelabour rupture of mambranes from $24+0$ weeks of gestation. green top guideline no. 73. BJOG 2019; 126: e152:166. DOI: $10.1111 / 1471-0528.15803$.

6. WHO recommendations on interventions to improve preterm birth outcomes; 2015. Downloaded from www.who.int/reproductivehealth/publications/ maternal_perinatal_health/preterm_birth_guideline

7. Bond DM, Middleton P, Levett KM, et al. Planned early birth versus expectant management for women with preterm prelabour rupture of membranes prior to 37 weeks' gestation for improving pregnancy outcome. Cochrane Database Syst Rev 2017; 3:CD004735.

8. Schucker JL, BM Mercer - Seminars in perinatology incidance of chorioamnionitis among patients with immediate delivery group versus expectant management, 1996

9. Tanir H, Sener T, Tekin N, Aksit A, Ardica N. Preterm premature rupture of membranes and neonatal outcomeprior to 34 weeks of gestation .2003 International Federation of Gynecology and Obstetrics. Published by Elsevier Science Irelan.

10. Quist-Nelson J, de Ruigh AA, Seidler AL, et al. Immediate Delivery Compared With Expectant Management in Late Preterm Prelabor Rupture of Membranes: An Individual Participant Data Meta $\neg$ analysis. ObstetGynecol 2018; 131:269.

11. Ramsey PS, Lieman JM, Brumfield CG et al.Chorioamnionitis increases neonatal morbidity in pregnancies complicated by preterm premature rupture of membranes American journal of obstetrics and gynecology, 2005 - Elsevier 